\title{
ELECTRIC FIELD TRANSIENTS OBSERVED BY THE HUYGENS PROBE IN THE ATMOSPHERE OF TITAN: ATMOSPHERIC ELECTRICITY PHENOMENA OR ARTEFACTS?
}

\author{
K. Schwingenschuh*, T. Tokano ${ }^{\dagger}$, I. Jernej*, H. U. Eichelberger*, \\ G. Prattes*, B. P. Besser*, V. Brown ${ }^{\ddagger}$, M. Hamelin ${ }^{\S}$, and the \\ PWA/HASI team
}

\begin{abstract}
During the first 35 close Titan flybys the Radio and Plasma Wave Science instrument (RPWS) aboard the CASSINI orbiter did not observe radio signals possibly associated with lightning in the atmosphere of Titan [Fischer et al., 2007, Geophys. Res. Lett., 34, L22104). The electric field sensors of the HUYGENS PWA instrument (permittivity, waves and altimetry) observed smooth variations as well as impulsive events varying with altitude during the descent of the probe in the atmosphere of Titan. While a part of the low frequency signals was explained as externally driven Schumann resonances, there is still a debate on the origin of the impulsive events. In order to differentiate natural atmospheric discharges from sources on the parachute or the probe the HUYGENS electric field data have been re-evaluated und combined with probe attitude and velocity. The correlation results indicate that atmospheric electricity phemonena are present in the atmosphere of Titan.
\end{abstract}

\footnotetext{
* Space Research Institute, Austrian Academy of Sciences, Schmiedlstrasse 6, A-8042 Graz, Austria † Institut fúr Geophysik und Meteorologie, Universität zu Köln, 50931 Köln, Germany

‡Instituto de Astrofisica de Andalucia, 18008 Granada, Spain

§ LATMOS, Université Versailles Saint-Quentin-en-Yvelines, CNRS, 78280 Guyancourt, France
} 
